\title{
Evaluation of Two Serological Screening Kits for Hepatitis C Virus Infection at the Regional Blood Transfusion Center of Ouagadougou, Burkina Faso
}

\author{
Arzouma Paul Yooda ${ }^{1,2}$, Koumpingnin Nebie1, Juliette Tranchot-Diallo³, Salam Sawadogo', \\ Moutanou Modeste Judes Zeye², Abdoul-Guaniyi Sawadogo', Abdou Azaque Zoure", \\ Dinanibè Kambire ${ }^{4}$, Serge Sawadogo', Seimbou Zalla1, Yetema Dieudonné Yonli', \\ Abibou Simpore ${ }^{1,2}$, Sibiri Nana1, Ashmed Chèickh Bachirou Nana1, Anita Pierrette Siritie ${ }^{4}$, \\ Fiffou Yougbare ${ }^{1,2}$, Sonia Ba-Nébhane Sontie ${ }^{1}$, Alain Konseybo', Jean Etienne Koanda ${ }^{1}$, \\ Oury Sanou ${ }^{1}$, Jacques Simpore ${ }^{2}$

\footnotetext{
${ }^{1}$ National Blood Transfusion Center (NBTC), Ouagadougou, Burkina Faso

${ }^{2}$ Laboratory of Molecular Biology and Genetics (LABIOGENE), Joseph KI-ZERBO University, Ouagadougou, Burkina Faso

${ }^{3}$ Training and Research Unit/Science and Technology (TRU/ST), Nazi-BONI University, Bobo-Dioulasso, Burkina Faso

${ }^{4}$ Research Institute in Health Sciences (IRSS/CNRST), Ouagadougou, Burkina Faso

Email: *yoodhaba@yahoo.fr
}

How to cite this paper: Yooda, A.P., Nebie, K., Tranchot-Diallo, J., Sawadogo, S., Zeye, M.M.J., Sawadogo, A.-G., Zoure, A.A., Kambire, D., Sawadogo, S., Zalla, S., Yonli, Y.D., Simpore, A., Nana, S., Nana, A.C.B., Siritie, A.P., Yougbare, F., Sontie, S.B.-N., Konseybo, A., Koanda, J.E., Sanou, O. and Simpore, J. (2020) Evaluation of Two Serological Screening Kits for Hepatitis C Virus Infection at the Regional Blood Transfusion Center of Ouagadougou, Burkina Faso. Advances in Infectious Diseases, 10, 216-227.

https://doi.org/10.4236/aid.2020.105019

Received: September 22, 2020

Accepted: October 30, 2020

Published: November 2, 2020

\begin{abstract}
Introduction: In Burkina Faso, screening for hepatitis $\mathrm{C}$ virus in blood donations is made using sensitive ELISA (Enzyme Linked Immuno Sorbent Assay) type kits. However, no confirmation of the positive results obtained with these kits is made before their notification to the blood donors due to the high costs of the confirmation kits of immunoblots type. Objective: Evaluate two rapid kits against one immunoblot kit in order to determine the most efficiency which will be proposed as an alternative for the confirmation of ELISA tests in the socio-economic context of Burkina Faso. Material and Methods: The study was carried out using a panel of 72 sera, of which 22 were positive for anti-HCV antibodies and 50 were negative. The sera were tested using the Monolisa ${ }^{\circledR} \mathrm{HCV}$ Ag-Ab ULTRA kit and confirmed with the DECISCAN HCV Plus kit. The panel was then tested with the SD BIOLINE HCV kit and the HCV TRI-DOT kit and the results obtained were evaluated against those of the DECISCAN HCV Plus kit used as "gold standard". Results: Compared to the DECISCAN HCV Plus kit, the HCV TRI-DOT kit exhibited a sensitivity and specificity of $100 \%$ and the SD BIOLINE HCV kit a sensitivity of $86.36 \%$ and a specificity of $100 \%$. Conclusion: Based on the results recorded by the HCV TRI-DOT kit, it would be best suited to the se-
\end{abstract}


Copyright $\odot 2020$ by author(s) and Scientific Research Publishing Inc. This work is licensed under the Creative Commons Attribution International License (CC BY 4.0).

http://creativecommons.org/licenses/by/4.0/

\section{Open Access}

ro-epidemiological context of blood donors from the National Blood Transfusion Center and could be proposed as an alternative for confirmation of ELISA tests.

\section{Keywords}

HCV, Sensitivity, Specificity, Burkina Faso

\section{Introduction}

The hepatitis C virus (HCV) is one of the major pathogens for which the world health organisation (WHO) recommends systematic screening of donated blood before any transfusion [1]. HCV infection is characterized by a long serological window (66 days) during which the virus replicates without the infected organism producing anti-HCV antibodies against it [2]. Thus, the residual risk of HCV transmission through blood transfusion would be due to this so-called silent period [2]. In order to ensure an optimal transfusion safety, WHO recommends the use of very sensitive screening kits, such as Enzyme-Linked ImmunoSorbent Assays (ELISA) in the biological qualification of blood donation [1]. Indeed, these combined antigen-antibody tests allow early detection of HCV, thus considerably reducing the residual risk linked to the serological window [3]. However, these tests turn out to be the source of a significant proportion of "false positives" results, especially with black blood donors [4]. There are several reasons for these false positives. These are in particular autoimmune diseases, viral infections, hepatic pathologies (cirrhosis), hypergammaglobulinemia, etc. [4]. These false positives not only lead to the destruction of a large quantity of blood products, the wrongful exclusion of a large number of blood donors in a context of scarcity of blood products, but also lead to stress for blood donors who find themselves in this situation. For these reasons, the results obtained by these tests require confirmation by molecular biology tests or by immunoblot type tests before blood donors are notified or before excluding them from donating blood. However, in developing countries (DCs), such confirmation is not always feasible due to the high cost of confirmatory testing.

In Burkina Faso, the management of "false positives" is all the more worrying given that HCV infection is endemic with an estimated prevalence of 3.4\% [5] in the general population and 4.4\% [6] among blood donors at Regional Blood Transfusion Center of Ouagadougou (RBTC/O).

Given the high-cost of the reference confirmation tests (molecular biology or immunoblot), the National Blood Transfusion Center (NBTC) of Burkina Faso uses rapid kits as an alternative to confirm the results obtained by ELISA kit of the fourth generation used in the biological qualification of blood donation.

This study aims to validate the diagnostic performances (sensitivity and specificity) of two rapid kits compared to an immunoblot kit in order to propose an 
algorithm for serological screening of HCV in blood donors in the epidemiological context of Burkina Faso.

\section{Material and Methods}

\subsection{Type and Scope of the Study}

This was a cross-sectional validation study of two serological screening tests for HCV which took place at RBTC/O between the period from January to March 2012.

The RBTC/O is one of the eight operational structures of the NBTC located in different regions of the country. The role of the NBTC is to coordinate the country's transfusion activities and the RBTC's missions are to collect, qualify and distribute labile blood products (LBP) to all health structures in their coverage areas. Since the operationalization of these RBTC, which began in 2005, the blood safety policy has continuously improved with the application of measures such as the medical selection of donors, the recruitment of unpaid voluntary donors from low-risk populations, the loyalty of blood donors, and the use of fourth-generation serological tests for the biological qualification of donations.

\subsection{Constitution of the Panel}

The samples included in the panel are serums from unpaid volunteer blood donors of both sexes aged from 18 to 60 years. Samples were collected on dry tubes from donors during blood donation, centrifuged at $4000 \mathrm{~g}$ for 5 minutes and collected serum were tested using the Monolisa ${ }^{\circledR}$ HCV Ag-Ab ULTRA kit (Bio-Rad, Marnes-la-Coquette-France) according to the strategy for the biological qualification of donations adopted at the NBTC. Then, samples were aliquoted and stored at $-80^{\circ} \mathrm{C}$ for the serotheque. We randomly selected 90 samples including 40 anti-HCV antibody positive and 50 negative samples, which we re-tested at DECISCAN HCV Plus kit (Bio-Rad, Marnes-la-Coquette-France) for confirmation. All 50 anti-HCV antibody negative serum to Monolisa ${ }^{\circledR} \mathrm{HCV}$ Ag-Ab ULTRA kit, were confirmed negative by DECISCAN HCV Plus kit. On the other hand, of the 40 anti-HCV antibody sero positive for Monolisa ${ }^{\circledR} \mathrm{HCV}$ Ag-Ab ULTRA kit, 22 were confirmed positive, nine were negative and nine others were unsuccessful (Figure 1). We then retained the 50 negative samples and the 22 DECISCAN HCV Plus positive samples (i.e. 72 samples) for our validation panel for the two rapid test kits.

\subsection{Carrying Out the Various Analyzes}

\subsubsection{The DECISCAN HCV plus Kit}

The DECISCAN HCV Plus kit (Bio-Rad, France) is an immunoblot validation test. It is a unit membrane test using an enzyme-immunoassay technique allowing the individualization of antibodies associated with HCV infection in human serum or plasma. 


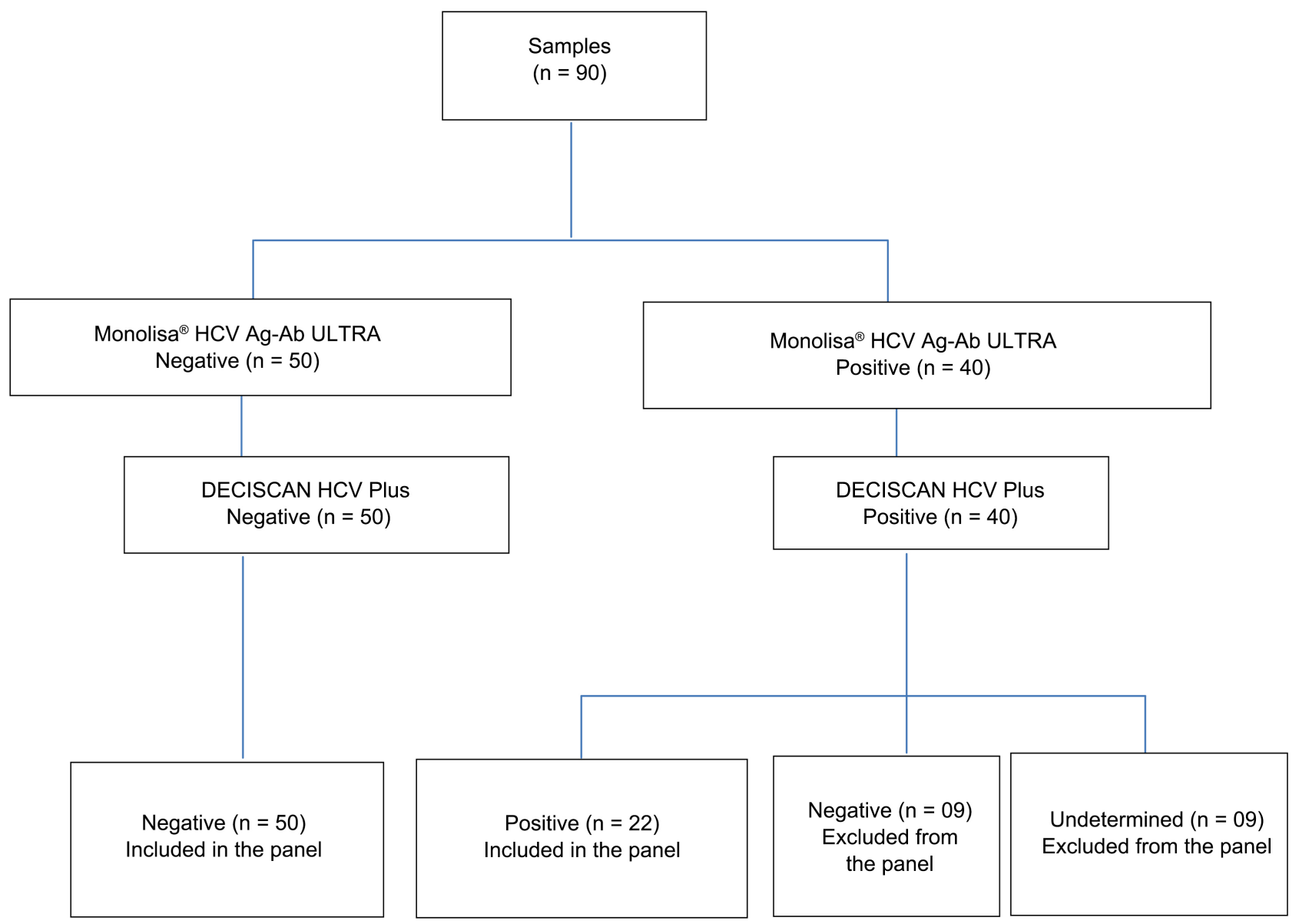

Figure 1. Algorithm for the inclusion of samples in the panel according to the results obtained with the DECISCAN HCV Plus.

The DECISCAN HCV Plus kit contains strips where the antigens (core, NS3, NS4 and NS5) are previously fixed. For analysis, the serum is first incubated with the strip. If the anti-HCV antibodies are present, they bind to the antigens fixed to the solid phase. A first wash is performed to remove excess serum and unbound antibodies. Then, alkaline phosphatase labeled anti-human IgG antibodies are added, which in turn will bind to the specific antibodies retained on the solid phase. A second wash is carried out to remove the unbound enzyme conjugate and finally the antigen-antibody complex is revealed by addition of the substrate.

\subsubsection{SD BIOLINE HCV Kit}

SD BIOLINE HCV kit (SD STANDARD DIAGNOSTICS, INC, Kyonggi-do Korea) a fourth-generation rapid test using an immunochromatographic principle for the qualitative detection of HCV specific antibodies in serum, plasma, or whole blood. The kit contains strips in which the recombinant antigens (Core, NS3, NS4, and NS5) are previously fixed (Table 1). The analytical procedure consists of depositing $10 \mu \mathrm{L}$ of serum in the deposit zone, then adding four drops of diluent. The sample migrates on the solid phase to the patient window phase $(\mathrm{T})$ and forms an antigen-antibody complex as manifested by line visible. 
Table 1. Characteristics of the kits evaluated according to the manufacturer.

\begin{tabular}{ccc}
\hline Characteristics of the kits & SD BIOLINE HCV kit & HCV TRI-DOT kit \\
\hline Maker & Standard Diagnostics, Inc. & J. Mitra \& Co. Pvt. Ltd \\
Principle & Immunochromatography & Immunofiltration \\
Antigens & (Core, NS3, NS4, NS5) & (Core, NS3, NS4, NS5) \\
Recommended & Serum, plasma, & Serum, plasma \\
biological sample & Whole blood & $2^{\circ} \mathrm{C}-8^{\circ} \mathrm{C}$ \\
Storage temperature & $1^{\circ} \mathrm{C}-30^{\circ} \mathrm{C}$ & Visual \\
Reading & Visual & $100 \%$ \\
Sensitivity & $100 \%$ & $99.8 \%$ \\
Specificity & $99.4 \%$ &
\end{tabular}

The results are read 15 minutes after the deposit according to the instructions provided by the manufacturer. The test is labeled with the letters $\mathrm{C}$ and $\mathrm{T}$ representing respectively the control band and the positive band of the test. The test is only valid if the control strip appears.

\subsubsection{HCV TRI-DOT Kit}

HCV TRI-DOT kit (J. Mitra \& Co. Pvt. Ltd., New Delhi-INDIA) is a fourth-generation rapid visual test using the principle of immuno-filtration for the qualitative detection of HCV specific antibodies in serum or plasma. The kit contains a unique combination of modified antigens from the core, NS3, and the NS4 and NS5 regions of HCV (Table 1). These antigens are immobilized on a porous immunofiltration membrane, which includes two test points, T1 and T2, and an additional point for quality control. When the sample infiltrates through the membrane, anti-HCV antibodies present, bind to antigens immobilized on the absorbent pad. Then a conjugate is added which binds to the Fc portion of $\mathrm{HCV}$-specific immunoglobulin G to give a distinct purple-pink separate dot in the test area. A color will appear at the control point after the patient's serum has been added regardless of the color that appears in the two test points ( $\mathrm{T} 1$ and T2), confirming that the reagents are working properly and that the procedure has been followed. The test run time is $5 \mathrm{~min}$.

\subsection{Statistical Analyzes}

The proportion of positive and negative results obtained was calculated for each test submitted for evaluation. The results were compared to those of DECISCAN HCV Plus kit in a $2 \times 2$ contingency table. Then, we calculated the sensitivity (true positives/true positives + false negatives) and specificity (true negatives/true negatives + false positive) for each test. We also determined the Youden index (sensitivity + specificity -1 ) to better establish the level of efficiency of the performance of the two tests evaluated compared to the gold standard. 


\subsection{Ethical Considerations}

The informed consent of blood donors was systematically obtained before any blood donation by signing the pre-donation medical interview sheet. The study received the approval of the internal scientific review committee of the NBTC. The anonymity and confidentiality of blood donors were respected.

\section{Results}

\subsection{Socio-Demographic Characteristics of Blood Donors Includ in the Study}

A total of 72 blood donors were included in this study. The age of the blood donors varied between 18 and 55 years with an average of $29.54+/-8.81$ years. The majority of blood donors were male $(69.44 \%)$ and were first-time donors (94.44\%) (Table 2).

\subsection{Serological Results Obtained with SD-BIOLINE HCV Kit and HCV TRI-DOT Kit}

All the 50 anti-HCV antibody negative to DECISCAN HCV Plus kit included in the study were tested negative with the SD BIOLINE HCV kit and with the HCV TRI-DOT kit. Similarly, all 22 serums HCV antibody positive to DECISCAN HCV Plus kit were tested positive to HCV TRI-DOT kit (Table 3). In contrast with SD BIOLINE HCV kit, 19 serums were positive and 03 negatives. No doubtful or indeterminate results were detected by these two tests.

Table 2. Socio-demographic characteristics of blood donors.

\begin{tabular}{ccc}
\hline Characteristics & Number $(\mathbf{n}=\mathbf{7 2})$ & Proportion (\%) \\
\hline Gender & 50 & 69.44 \\
Male & 22 & 30.56 \\
Female & & \\
Age groups & 13 & 18.05 \\
$<20$ ans & 35 & 48.61 \\
$20-29$ ans & 9 & 12.50 \\
$30-39$ ans & 11 & 15.28 \\
$40-49$ ans & 4 & 5.56 \\
$\geq 50$ ans & 4 & 9.56 \\
Donors status & & \\
First time donors & & \\
Repeat donors & 48.44
\end{tabular}


Table 3. Serological results obtained with SD BIOLINE HCV kit and HCV TRI-DOT kit.

\begin{tabular}{ccc}
\hline & & DECISCAN HCV Plus \\
\cline { 3 - 3 } & & HCV + HCV - \\
\hline SD BIOLINE HCV & HCV+ & 1900 \\
& HCV- & 0350 \\
HCV TRI-DOT & HCV+ & 2200 \\
& HCV- & 0050 \\
\hline
\end{tabular}

\subsection{Performance of SD B IOLINE HCV Kit and HCV TRI-DOT Kit}

Compared to the gold standard, the SD BIOLINE HCV kit has a sensitivity of $86.36 \%$ and a specificity of $100 \%$. The HCV TRI-DOT kit showed a sensitivity and specificity of $100 \%$. Table 4 gives the different intrinsic performances (sensitivity, specificity) and the levels of effectiveness (Youden index) of SD BIOLINE HCV kit and HCV TRI-DOT kit compared to the gold standard. Figure 2 shows Receiver Operating Characteristics (ROC) curve comparing the performance of SD BIOLINE HCV and HCV TRI-DOT tests in blood donors

\section{Discussion}

Our work aimed to evaluate two serologic tests for HCV infection (SD BIOLINE HCV, HCV TRI-DOT) compared to the DECISCAN HCV Plus kit to propose an alternative and inexpensive algorithm for HCV serological screening in our socio-economic context. Both tests were evaluated using a panel of serums collected from blood donors in RBTC of Ouagadougou. We chose to evaluate these two tests because they are very easy to use, provide results in less than $30 \mathrm{~min}$ and are less expensive, especially in resources limited contexts. They do not require highly qualified laboratory technicians, or specific laboratory equipment [7]. In addition, these kits were already present in the list of tests marketed and used in Burkina Faso for HCV screening. But, immunoblot kits such as DECISCAN HCV PLUS use enzyme-immunoassay technics and require relatively expensive laboratory equipment, reagents and relatively long handling times.

The study showed that SD BIOLINE HCV kit and HCV TRI-DOT kit both have a specificity of $100 \%$ and sensitivities of $86.36 \%$ and $100 \%$ respectively (Table 4). As in our study, several other studies report that rapid screening tests for anti-HCV antibodies generally have good specificity (100\%) but not all have good sensitivities [8] [9] [10]. The Youden index was 1 for the HCV TRI-DOT kit indicating a perfect detection capacity of both HCV positive and negative subjects. On the other hand, the Youden index SD BIOLINE HCV kit was 0.86 showing that about $14 \%$ of the samples were not properly detected (Table 4 ). These results are in agreement with several other studies that report that rapid tests for HCV are generally low in sensitivity in the African context. For example, an international multicenter study evaluating eight (8) rapid kits involving 


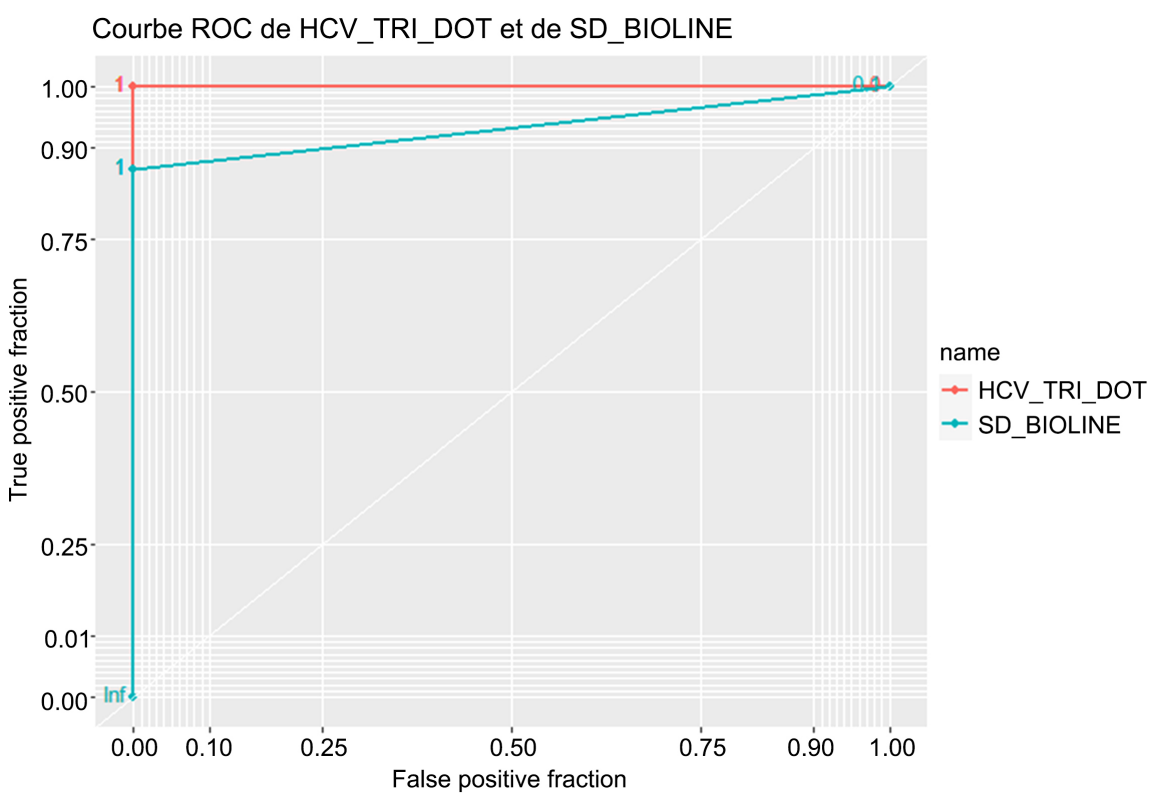

Figure 2. Receiver Operating Characteristics (ROC) curve comparing the performance of SD BIOLINE HCV and HCV TRI-DOT tests in blood donors.

Table 4. Performances of SD BIOLINE HCV and HCV TRI-DOT.

\begin{tabular}{cccc}
\hline Kits & Sensitivity (\%) & Specificity (\%) & Youden Index \\
\hline HCV TRI-DOT & $100 \%(95 \% \mathrm{CI})$ & $100 \%(95 \% \mathrm{CI})$ & 1 \\
SD BIOLINE HCV & $86.36 \%(95 \% \mathrm{CI})$ & $100 \%(95 \% \mathrm{CI})$ & 0.86 \\
\hline
\end{tabular}

51 blood transfusion centers in 17 African countries reported that the overall sensitivity of these tests was $63.7 \%$ with variations ranging from $43 \%$ to $100 \%$ [10]. Similarly, in Burkina Faso, three rapid kits evaluated using serums from RBTC/O blood donors had low sensitivities ranging from 33\% to $47 \%$ [7]. These different results show that rapid tests have great variability in performance, especially in the African context, as shown in this study. This situation could be explained by multiple factors, in particular the genetic variability of the viral strains, the seroprevalence of the infection in the population, the conditions of transport and storage of the screening kits, which are likely to influence the sensitivity of the tests [2] [11] [12]. Indeed, HCV has six different genotypes with great genetic diversity. Thus, the ability of each test to detect the different genotypes circulating in each geographical locality depends strongly on the nature and types of antigens that make it up [8] [9]. For this reason, the choice of commercial tests for a particular geographic area should always be preceded by adequate assessment with samples collected locally to determine the performance (sensitivity and specificity) of the test in this specific context, which may differ from those given by the manufacturer [7].

The poor performance of SD BIOLINE HCV kit (sensibility to $86.36 \%$ and index Youden 0.86) means that this test would lead to false negatives when used 
in the context of Burkina Faso. It therefore does not guaranteed optimal transfusion safety. Many donors would be screened false negative because of a lack of sensitivity, which would endanger the health of recipients. This test would be better indicated for the diagnosis of HCV infection in a clinical setting. The specificity of $100 \%$ obtained with SD BIOLINE HCV kit in our study is consistent with that given by the manufacturer and is similar to those found in other studies [7] [9]. In contrast, the sensitivity of $86.36 \%$ obtained in the study is lower than that provided by the manufacturer (99.4\%). Other authors, Laperche et al. (2013) and Ouedraogo et al. (2019) had also reported respectively in their studies of sensitivities of $72.2 \%$ and of $33.87 \%$, significantly lower than those provided by the manufacturer (99.4\%) [7] [10]. However, in a study conducted in India, the SD BIOLINE HCV kit had a sensitivity of $100 \%$ on a panel of serums from patients seen in a hepato-gastroenterology department and $97.4 \%$ on another panel of serums from blood donors [9]. These different results show that the performance of the tests can vary according to the nature of the population studied.

HCV TRI-DOT kit exhibits good sensitivity (100\%) and good specificity (100\%) (Table 4) concerning the serums collected locally in Burkina Faso. This performance means that both false negative and false positive results would be minimized by this test. It would ensure better transfusion safety and could be used as a second line alternative to immunoblot confirmatory tests for the confirmation of results obtained by ELISA kit in blood transfusion centers. Besides, its cost is relatively affordable and its use is less demanding in terms of technical platform compared to immunoblot kits validation. The sensitivity (100\%) and specificity (100\%) obtained by the HCV TRI-DOT kit were respectively in line with the manufacturer's specifications with $100 \%$ and $99.8 \%$ respectively. Daniel et al. (2005) had also reported a good sensitivity of $99.3 \%$ and a good specificity of $99 \%$ of HCV TRI-DOT kit in a study evaluating this test compared to conventional ELISA kits [8]. In the same study, Daniel et al. (2005) have also shown that this test was less sensitive to certain genotypes circulating in India including genotypes 1 and 3 [8]. However, these authors admit, as shown in our study, that HCV TRI-DOT kit provides excellent performance and competes in terms of sensitivity with ELISA kits [8].

Given the results obtained, we proposed a serological screening algorithm adapted to the socioeconomic context of Burkina Faso (Figure 3). The objective of this algorithm is to reduce as much as possible the use of confirmatory immunoblot tests while ensuring the reliability of the results. In terms of blood transfusion, the parameter sought is sensitivity because it guarantees the test's ability to detect the weakest positive samples. However, in order not to wrongly exclude donors from donating blood and also to return the results to blood donors who wish to know their serological status, it is advisable to confirm them by a test with good specificity. To do this, we have selected the HCV TRI-DOT kit with regard to its performance to offer an algorithm sequentially combining an 


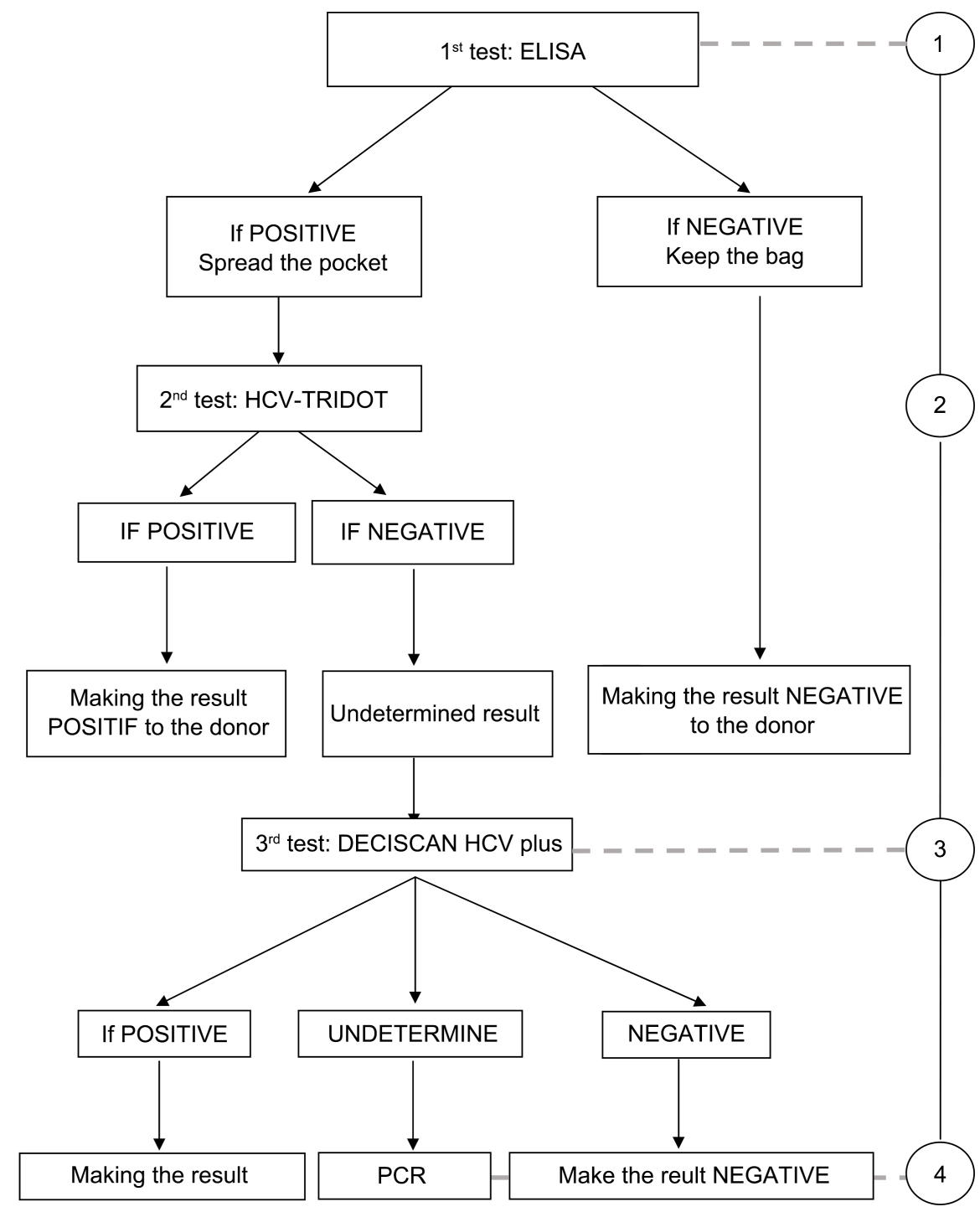

Figure 3. Proposal for an algorithm for serological screening for HCV including HCV TRI-DOT kit.

enzyme immunoassay (Monolisa ${ }^{\circledR} \mathrm{HCV}$ Ag-Ab ULTRA) as a first-line test, a rapid test (HCV TRI-DOT HCV) as a second-line and an immunoblot test (DECISCAN HCV Plus) to test any cases of discrepancies that may be encountered between the first two tests. In this case, any positive result on the first-line test (Monolisa ${ }^{\circledR} \mathrm{HCV}$ Ag-Ab ULTRA) and positive in the second test (HCV TRI-DOT) will be considered positive and the result will be declared positive to the donor and the donor will be excluded from donating blood. On the other hand, a positive result on the first-line test and negative for HCV TRI-DOT kit will be considered "indeterminate" and a control by a third immunoblot test is necessary to conclude.

\section{Conclusion}

This study showed that not all commercial screening tests for HCV infection 
present the same diagnostic performance in the epidemiological context of Burkina Faso. This implies the imperative need to evaluate the tests before their use for optimal transfusion safety. Besides, our study made it possible to propose an alternative screening algorithm adapted to the socio-economic context of Burkina Faso. This algorithm combines a test capable of ensuring optimal transfusion safety with a high sensitivity to a second test to guarantee reliable results to donors with a specificity of $100 \%$.

\section{Conflicts of Interest}

The authors declare no conflicts of interest regarding the publication of this paper.

\section{References}

[1] OMS. (2010) Dépistage des infections transmissibles par le sang. Recommandations.

[2] Tagny, C.T., Mbanya, D., Murphy, E.L., Lefrère, J.-J. and Laperche, S. (2014) Screening for Hepatitis C Virus Infection in a High Prevalence Country by an Antigen/Antibody Combination Assay versus a Rapid Test. Journal of Virological Methods, 199, 119-123. https://doi.org/10.1016/j.jviromet.2014.01.002

[3] Laperche, S., Le Marrec, N., Girault, A., Bouchardeau, F., Servant-Delmas, A., Maniez-Montreuil, M., Gallian, P., Levayer, T., Morel, P. and Simon, N. (2005) Simultaneous Detection of Hepatitis C Virus (HCV) Core Antigen and Anti-HCV Antibodies Improves the Early Detection of HCV Infection. Journal of Clinical Microbiology, 43, 3877-3883. https://doi.org/10.1128/JCM.43.8.3877-3883.2005

[4] Vo, M.T., Bruhn, R., Kaidarova, Z., Custer, B.S., Murphy, E.L. and Bloch, E.M. (2016) A Retrospective Analysis of False-Positive Infectious Screening Results in Blood Donors. Transfusion, 56, 457-465. https://doi.org/10.1111/trf.13381

[5] Meda, N., Tuaillon, E., Kania, D., Tiendrebeogo, A., Pisoni, A., Zida, S., Bollore, K., Medah, I., Laureillard, D. and Moles, J.P. (2018) Hepatitis B and C Virus Seroprevalence, Burkina Faso: A Cross-Sectional Study. Bulletin of the World Health Organization, 96, 750. https://doi.org/10.2471/BLT.18.208603

[6] Yooda, A.P., Sawadogo, S., Soubeiga, S.T., Obiri-Yeboah, D., Nebie, K., Ouattara, A.K., Diarra, B., Simpore, A., Yonli, Y.D. and Sawadogo, A.-G. (2019) Residual Risk of HIV, HCV, and HBV Transmission by Blood Transfusion between 2015 and 2017 at the Regional Blood Transfusion Center of Ouagadougou, Burkina Faso. Journal of Blood Medicine, 10, 53. https://doi.org/10.2147/JBM.S189079

[7] Ouedraogo, H.G., Ouandaogo, H.S., Zida, S., Compaoré, T.R., Sagna, T., Sawadogo, S., Kouanda, S., Traore, Y. and Barro, N. (2019) Evaluation of Three Commercialized Rapid Point-of-Care Tests for Detection of Anti-Hepatitis C Virus Antibodies in Burkina Faso. African Journal of Microbiology Research, 13, 710-715.

[8] Daniel, H.D.J., Abraham, P., Raghuraman, S., Vivekanandan, P., Subramaniam, T. and Sridharan, G. (2005) Evaluation of a Rapid Assay as an Alternative to Conventional Enzyme Immunoassays for Detection of Hepatitis C Virus-Specific Antibodies. Journal of Clinical Microbiology, 43, 1977-1978. https://doi.org/10.1128/JCM.43.4.1977-1978.2005

[9] Mane, A., Sacks, J., Sharma, S., Singh, H., Tejada-Strop, A., Kamili, S., Kacholia, K., Gautam, R., Thakar, M. and Gupta, R.S. (2019) Evaluation of Five Rapid Diagnostic Tests for Detection of Antibodies to Hepatitis C Virus (HCV): A Step towards 
Scale-Up of HCV Screening Efforts in India. PLOS ONE, 14, e0210556.

https://doi.org/10.1371/journal.pone.0210556

[10] Laperche, S. (2013) Multinational Assessment of Blood-Borne Virus Testing and Transfusion Safety on the African Continent. Transfusion, 53, 816-826. https://doi.org/10.1111/j.1537-2995.2012.03797.x

[11] Ndjoyi-Mbiguino, A. and Bélec, L. (2005) Évaluation de trousses de dépistage de l'infection à VIH au Gabon. Cahiers d'études et de recherches francophones/Santé, 15, 23-29.

[12] Delwart, E., Slikas, E., Stramer, S.L., Kamel, H., Kessler, D., Krysztof, D., Tobler, L.H., Carrick, D.M., Steele, W. and Todd, D. (2012) Genetic Diversity of Recently Acquired and Prevalent HIV, Hepatitis B Virus, and Hepatitis C Virus Infections in US Blood Donors. Journal of Infectious Diseases, 205, 875-885.

https://doi.org/10.1093/infdis/jir862 